\title{
Identificazione del contributo alla spermatogenesi del gene $X$-linked BCORL1
}

\author{
Carmelo Gusmano ${ }^{1} \cdot$ Aldo E. Calogero ${ }^{1}$
}

Accettato: 6 marzo 2021 / Pubblicato online: 8 settembre 2021

(c) The Author(s) 2021

\section{Commento a:}

Human X chromosome exome sequencing identifies BCORL1 as contributor to spermatogenesis.

C. Lu, Y. Zhang, Y. Qin, Q. Xu, R. Zhou, Y. Cui, Y. Zhu, X. Zhang, J. Zhang, X. Wei, M. Wang, B. Hang,

J.H. Mao, A.M. Snijders, M. Liu, Z. Hu, H. Shen, Z. Zhou, X. Guo, X. Wu, X. Wang, Y. Xia. J Med Genet (2021) 58(1):56-65

L'infertilità ha un'elevata prevalenza nei paesi industrializzati, pari a circa un settimo delle coppie in età riproduttiva. In circa il $50 \%$ dei casi, la sua eziologia è riconducibile a un fattore maschile, da solo o in associazione a quello femminile. L'infertilità maschile è dovuta a molteplici cause $\mathrm{e}$, tra queste, quelle di natura genetica sono presenti in circa il $15 \%$ dei casi. Le cause genetiche ad oggi maggiormente note sono le anomalie del cariotipo, le mutazioni di CFTR nell'agenesia dei dotti deferenti e le microdelezioni del cromosoma Y [1]. Per contro, le alterazioni monogeniche Xlinked non sono apparentemente annoverate tra queste cause, sebbene questo cromosoma sia coinvolto nel processo di spermatogenesi. Rispetto alle piccole dimensioni del cromosoma $\mathrm{Y}$, il cromosoma X contiene circa il 5\% di tutto il DNA umano. I maschi hanno una sola copia del cromosoma $\mathrm{X}$ e, quindi, $5 \%$ del loro genoma è presente in emizigosi. Pertanto, le mutazioni de-novo dei geni che mappano sul cromosoma X, non essendo compensate dal cromosoma omologo, manifestano sempre il fenotipo patologico. Queste evidenze rendono lo studio dei geni X-linked coinvolti nella spermatogenesi un buon target di approfondimento delle cause di infertilità maschile.

\footnotetext{
$凶$ A.E. Calogero

acaloger@unict.it

1 Dipartimento di Medicina Clinica e Sperimentale, Università di Catania, Catania, Italia
}

Nonostante il ruolo del cromosoma X nella spermatogenesi sia stato confermato ormai da qualche decennio, i pattern di espressione e il potenziale impatto sull'infertilità maschile dei geni che mappano in questo cromosoma non sono ben conosciuti. Per meglio approfondire questo aspetto, Lu e colleghi hanno valutato la presenza di alterazioni di geni localizzati sul cromosoma X su una coorte di pazienti infertili e soggetti fertili. I primi sono stati selezionati dopo attenta valutazione anamnestica, accurato esame obiettivo ed esecuzione di spermiogramma, ecografia testicolare, valutazione endocrina, esame del cariotipo e valutazione delle microdelezioni Yq [2]. Pazienti con storia di criptorchidismo, trauma vascolare, orchite, ostruzione dei dotti deferenti, vasectomia, anomalie del cariotipo o presenza di microdelezioni Yq sono stati esclusi dallo studio [2]. Il gruppo di controllo è stato selezionato dal registro delle gravidanze, all'interno della stessa struttura ospedaliera, selezionando i partner di donne al primo trimestre di gravidanza e che poi hanno partorito neonati sani [2].

Partendo da una casistica di 1333 pazienti infertili e 1141 controlli fertili, gli autori dello studio hanno effettuato il sequenziamento degli esoni del cromosoma $\mathrm{X}$ su una popolazione di 96 pazienti con alterazione dei parametri spermatici di natura idiopatica e di 96 controlli sani, al fine di ricercare variazioni del singolo nucleotide (SNV) patologiche, ovvero presenti in meno dell' $1 \%$ della popolazione [2]. I risultati dello studio hanno evidenziato una diversa distribuzione di frequenza di SNV di geni che mappano sul cromosoma $\mathrm{X}$ tra pazienti infertili e uomini fertili. Queste SNV riguardano i geni ARMCX4, BCORL1, H2BFWT e MTAP7D3 [2]. Per valutare se essi abbiano un ruolo nel mantenimento delle cellule germinali staminali, gli autori hanno identificato geni omologhi nel topo (ad esclusione del gene H2BFWT) e hanno valutato la loro funzione sulla spermatogenesi silenziandone l'espressione nelle cellule germinali staminali di 
topo (modello knock-out, KO). In modo molto interessante, i risultati di questi esperimenti hanno dimostrato che il silenziamento di Armcx4 aumenta significativamente il numero di cellule germinali, il KO per Mtap $7 d 3$ diminuisce progressivamente il numero totale delle cellule germinali dopo 3, 5 e 7 giorni, e che il KO per Bcorll riduce significativamente il numero delle cellule germinali rispetto ai controlli dopo 7 giorni. Lo studio ha inoltre dimostrato come anche i topi maschi KO per Bcorll siano infertili e la mancanza di questo gene alteri la guaina mitocondriale. Pertanto, le SNV riscontrate a carico dei geni X-linked nel gruppo dei pazienti infertili sono verosimilmente responsabili dell'alterazione della spermatogenesi presente in questi ultimi.

In conclusione, le mutazioni di alcuni geni che mappano sul cromosoma $X$ sembrano svolgere un ruolo importante nella patogenesi delle alterazioni della spermatogenesi. Gli autori, impiegando un approccio sperimentale integrato, hanno trovato che le mutazioni dei geni X-linked BCORL1, MAP7D3 e ARMCX4 si associano a infertilità nell'uomo. Inoltre, questi dati forniscono una possibile spiegazione su come i geni X-linked possano alterare la spermatogenesi, aprendo strade innovative per identificare nuovi geni coinvolti, se mutati, nella patogenesi dell'infertilità maschile [2].
Funding Note Open access funding provided by Università degli Studi di Catania within the CRUI-CARE Agreement.

Nota della casa editrice Springer Nature rimane neutrale in riguardo alle rivendicazioni giurisdizionali nelle mappe pubblicate e nelle affiliazioni istituzionali.

Open Access This article is licensed under a Creative Commons Attribution 4.0 International License, which permits use, sharing, adaptation, distribution and reproduction in any medium or format, as long as you give appropriate credit to the original author(s) and the source, provide a link to the Creative Commons licence, and indicate if changes were made. The images or other third party material in this article are included in the article's Creative Commons licence, unless indicated otherwise in a credit line to the material. If material is not included in the article's Creative Commons licence and your intended use is not permitted by statutory regulation or exceeds the permitted use, you will need to obtain permission directly from the copyright holder. To view a copy of this licence, visit http://creativecommons.org/licenses/by/4.0/.

\section{Bibliografia}

1. Krausz C (2011) Male infertility: pathogenesis and clinical diagnosis. Best Pract Res Clin Endocrinol Metab 25:271-285

2. Lu C, Zhang Y, Qin Y et al (2021) Human X chromosome exome sequencing identifies BCORL1 as contributor to spermatogenesis. J Med Genet 58(1):56-65 